\title{
Glucocorticoid receptor gene (NR3C1) promoter is hypermethylated in Thai females with major depressive disorder
}

\author{
M. Nantharat ${ }^{1,2,3}$, T. Wanitchanon ${ }^{2}$, M. Amesbutr ${ }^{2}$, R. Tammachote ${ }^{1,3}$ and \\ V. Praphanphoj ${ }^{2}$ \\ 'Biological Sciences Program, Faculty of Science, Chulalongkorn University, \\ Bangkok, Thailand \\ ${ }^{2}$ Center for Medical Genetics Research, Rajanukul Institute, Bangkok, Thailand \\ ${ }^{3}$ Human Genetics Research Group, Department of Botany, Faculty of Science, \\ Chulalongkorn University, Bangkok, Thailand \\ Corresponding author: R. Tammachote \\ E-mail: Ratchaneekorn.T@chula.ac.th \\ Genet. Mol. Res. 14 (4): 19071-19079 (2015) \\ Received August 17, 2015 \\ Accepted October 23, 2015 \\ Published December 29, 2015 \\ DOI http://dx.doi.org/10.4238/2015.December.29.15
}

ABSTRACT. Major depressive disorder (MDD) has been associated with the stress response in the brain, which is controlled by the hypothalamicpituitary-adrenal (HPA) axis. This HPA negative feedback mechanism acts via the activation of glucocorticoid receptor, which is encoded by the $N R 3 C 1$ gene. The methylation status at the promoter of $N R 3 C 1$ has been correlated with traumatic experiences in early life, which develop into mental disorder. The aim of this study was to examine the potential associations between the methylation status of NR3C1 promoter, gene expression, blood plasma cortisol levels, and adulthood MDD. The study was conducted with 29 MDD patients (9 males, 20 females) and 33 normal individuals ( 7 males, 26 females). Bisulfite pyrosequencing on $7 \mathrm{CpG}$ dinucleotides in the region showed significantly higher methylation levels at the CpG7 in MDD patients. When separated by gender, the methylation levels differed significantly in females, but not in males. No significant 
differences between NR3C1 gene expression level and plasma cortisol levels of MDD patients and normal controls were observed. These data suggest that higher levels of methylation at the NR3C1 promoter may be associated with MDD in a gender-specific manner.

Key words: DNA methylation; MDD; HPA; NR3C1

\section{INTRODUCTION}

Major depressive disorder (MDD) is a common mental disorder affecting more than 350 million people worldwide (World Health Organization, 2012). Nevertheless, the pathological mechanism of MDD has not yet been elucidated. Previous research indicates that MDD may be associated with the stress response in the brain as in other stress-related disorders (Weaver et al., 2004; Oberlander et al., 2008; McGowan et al., 2009; Perroud et al., 2011). The stress response is primarily mediated by the hypothalamic-pituitary-adrenal (HPA) axis, whichis regulated by a negative feedback mechanism of the endocrine stress response to control cortisol levels (Meaney, 2001; Moser et al., 2007). The HPA negative feedback effect is exerted via the activation of glucocorticoid receptor (GR), also known as nuclear receptor subfamily 3, group C, member 1 (NR3C1), by high cortisol levels (Jacobson and Sapolsky, 1991; de Kloet et al., 2005). In humans, the GR protein is encoded by the NR3C1 gene on chromosome 5q31-q32 (Hollenberg et al., 1985; Francke and Foellmer, 1989). Studies in both animal models (Meaney, 2001; Howell and Muglia, 2006) and humans (Oberlander et al., 2008; Bet et al., 2009; McGowan et al., 2009; Perroud et al., 2011) have shown that the stress response is related to the NR3C1 gene and that the HPA axis plays a role in depression or depression-like conditions as well as in the etiology of many stressrelated behaviors. Inhibited HPA negative feedback activity is furthermore known to be associated with high circulating cortisol levels in MDD patients (Pariante et al., 2004).

The findings of several recent studies indicate that an epigenetic mechanism, which is partly influenced by environmental factors, regulates $N R 3 C 1$ expression. Research in rat models showed that increased DNA methylation of the nerve growth factor-inducible protein A (NGFI-A) binding site located in the NR3C1 gene exon $1_{7}$ promoter correlates with low maternal care (Weaver et al., 2004). This epigenetic process led to decreased NR3C1 expression and in turn impaired the negative feedback mechanism (de Kloet et al., 2005). These findings from animal studies were further supported by studies in human subjects: the DNA methylation status at a similar site (exon 1F in humans) in newborn cord blood was found to be sensitive to prenatal maternal mood disorders, and DNA methylation levels were found to relate to salivary cortisol levels (Oberlander et al., 2008). A study in suicidal victims with a history of childhood abuse showed high NR3C1 methylation in the hippocampus, which correlated with decreased NR3C1 expression levels (McGowan et al., 2009). The severity and type of childhood maltreatment was furthermore shown to be positively associated with NR3C1 methylation (Perroud et al., 2011). These findings suggest a relationship between traumatic experience in early life, which may develop into mental disorders, and increased NR3C1 methylation status. The role of DNA methylation status of the human NGFI-A binding site in NR3C1 expression in adult MDD patients, however, remains to be experimentally proven.

The aim of this study was therefore to examine the association between glucocorticoid receptor expression and methylation status of the $N R 3 C 1$ gene exon $1 \mathrm{~F}$ promoter region and adulthood MDD. The cortisol levels in the blood plasma of MDD patients were also assessed for potential correlation with the methylation status. 


\section{MATERIAL AND METHODS}

\section{Subjects}

The ethical approval request form, including Thai versions of the study protocol, patient information sheet, and informed consent form, were approved by The Ethical Review Committee for Research in Human Subjects (Mental Health and Psychiatry), Ministry of Public Health, Thailand. All participants were recruited from hospitals around Thailand: Suanprung and Maharaj Nakorn Chiang Mai Hospitals in the north; Loei Rajanagarindra Psychiatric Hospital in the north-east; Ramathibodi Hospital, Srithanya Hospital, and Prasat Neurological Institute in central Thailand; and Songklanagarind Hospital in the south. A total of 62 subjects including 29 MDD patients ( 9 males and 20 females; average ages $48.63 \pm 8.43$ years and $48.00 \pm 12.08$ years, respectively) and 33 normal individuals ( 7 males and 26 females; average ages $41.14 \pm 7.71$ years and $42.92 \pm 7.82$ years, respectively) were included in this study. All MDD subjects were diagnosed by psychiatrists and had not been treated with antidepressants. Normal controls were evaluated using a Thai language version of the Mini-International Neuropsychiatric Interview (Thai MINI) and showed no signs or symptoms of depression.

Peripheral blood samples were obtained from all 62 participants in the morning (average time $10.13 \pm 0.37$ a.m.) and kept in EDTA-containing tubes. All blood samples were immediately centrifuged at $515 \mathrm{~g}$ for 10 minutes to separate plasma serum, buffy coat, and red blood cells. Cortisol analysis was performed on plasma serum and genomic DNA and total RNA were extracted from the buffy coat using the illustra ${ }^{T M}$ blood genomic Prep Mini Spin Kit (GE Healthcare, UK) and the QIAamp ${ }^{\circledR}$ RNA Blood Mini Kit (Qiagen, Germany), respectively, according to the manufacturer instructions.

\section{Bisulfite pyrosequencing DNA methylation analysis}

Genomic DNA was sent to EpigenDx Inc. (http://www.epigendx.com) for DNA methylation status analysis according to standard procedures with primer sets developed by EpigenDx using two approaches. Briefly, the human long interspersed nucleotide elements (LINE-1) assay was used to estimate the global DNA methylation as previous described (Yang et al., 2004) using the PyroMark LINE-1 kit (Biotage-Qiagen, Uppsala, Sweden). In parallel, the genomic DNA of the same subjects was used for analysis of the methylation status in the exon $1 \mathrm{~F}$ promoter region of the NR3C1 gene by sodium bisulfite treatment using the EpiTect Bisulfite Kit (Qiagen, Germany) according to the manufacturer instructions. The PCR primers and conditions, as well as pyrosequencing primers were as described by Oberlander et al. (2008). The PCR products were purified and sequenced using a PyroMark MD System (Biotage) to analyze the DNA methylation status. In-house methylation samples, which exhibit low and high methylation status, were used as controls for the methylation analysis. The methylation percentage at each $\mathrm{CpG}$ site of both global and NR3C1 gene-specific methylation analyses were determined using the Pyro Q-CpG software (Biotage).

A portion of the exon $1 \mathrm{~F}$ promoter region of theNR3C1 gene containing seven $\mathrm{CpG}$ sites (Figure 1) was analyzedon CpGs 5 and 6 representing the NGFI-A binding site that regulates gene transcription. All seven CpG sites correspond to CpGs 33-39described by McGowan et al. (2009) and are located upstream to the CpGs 1-5 reported by Oberlander et al. (2008). The average methylation percentage of all CpG sites was calculated and used to analyze the methylation status of each sample. 


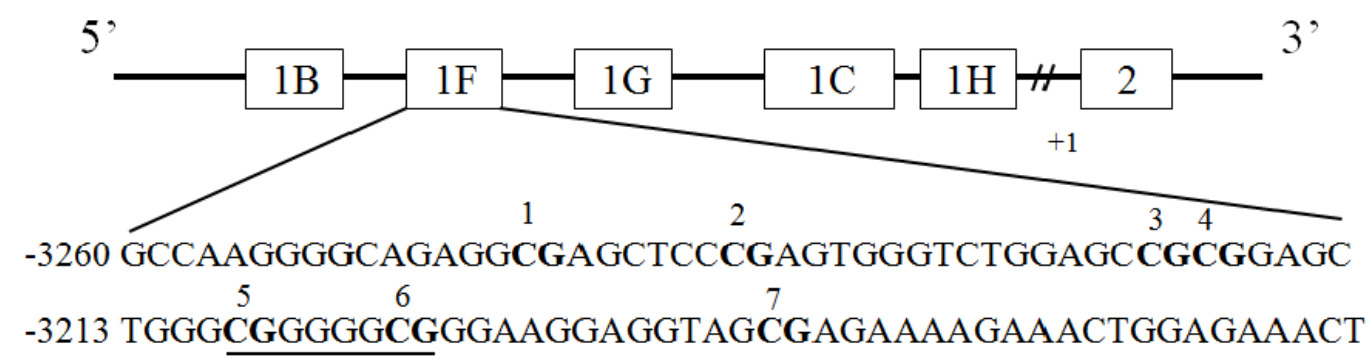

Figure 1. Schematic representation of the human NR3C1 gene exon $1 \mathrm{~F}$ promoter region methylation from nucleotide -3260 to -3167 (numbering is relative to the translational start site considered +1 ). The depiction is based on the previously characterized 5' end of the human NR3C1 gene promoter region (Oberlander et al., 2008; McGowan et al., 2009), which contains multiple first exons. The $7 \mathrm{CpG}$ dinucleotides in exon $1 \mathrm{~F}$ analyzed by bisulfite pyrosequencing are in bold fonts. The NGFI-A transcription factor binding site including $\mathrm{CpG} 5$ and $\mathrm{CpG} 6$ is underlined.

\section{Quantitative RT-PCR analysis of NR3C1 expression}

Extracted RNA was reverse transcribed using iScript ${ }^{\mathrm{TM}}$ Reverse Transcription Supermix (Bio-Rad,USA).Real-time PCR was performed to quantitate the mRNA expression levels in MDD patients $(N=29)$ and control subjects $(N=33)$. Real-time $P C R$ was carried out in triplicate using $2 X$ SYBR Green PCR Master Mix (QuantiTect ${ }^{\circledR}$ SYBR $^{\circledR}$ Green PCR Kit, Qiagen, Germany) according to the manufacturer instructions. NR3C1 primers used were as follows: sense, 5'-CTC CTC AAC AGC AAC AAC AG-3' and antisense, 5'-CAA TCA TTC CTT CCA GCA CAT AG-3'. GAPDH was used as an internal control and was amplified using primers described by McGowan et al. (2009). The qPCR cycling conditions were as follows: an initial activation step of $95^{\circ} \mathrm{C}(15 \mathrm{~min})$, followed by 35 cycles of denaturation at $95^{\circ} \mathrm{C}$ for $30 \mathrm{sec}$, annealing at $58^{\circ} \mathrm{C}$ for $30 \mathrm{sec}$, and elongation at $72^{\circ} \mathrm{C}$ for $30 \mathrm{sec}$. Relative gene expression levels of $N R 3 C 1$ were calculated using the $2^{-\Delta \Delta C t}$ method (Pfaffl, 2001).

\section{Cortisol level analysis}

Cortisol levels in blood plasma samples were quantified using the chemiluminescence immunoassay (CLIA) method, and were analyzed by the PCT Laboratory Services Co., Ltd. (Thailand).

\section{Statistical analysis}

DNA methylation statuses, relative gene expression levels, and cortisol levels in MDD patients and controls were compared using the unpaired $t$-test $\left(\right.$ Microsoft ${ }^{\circledR}$ Excel $\left.{ }^{\circledR}\right)$. Differences with $\mathrm{P}<0.05$ were considered statistically significant.

\section{RESULTS}

\section{Methylation status of NR3C1 in Thai MDD patients}

Methylation statuses at each of the seven $\mathrm{CpG}$ sites in the NR3C1 gene exon $1 \mathrm{~F}$ promoter in blood samples of Thai MDD patients and normal controls are shown in Figure 2. Global DNA 
methylation analysis, represented by the LINE-1 methylation status at four sites, showed no significant differences between normal controls and MDD patients $(P>0.05$; Figure $2 A)$. The results of the methylation status analysis in the NR3C1gene showed significant differences between MDD patients and normal controls only at $\mathrm{CpG} 7(\mathrm{P}<0.05)$ (Figure 2B). Interestingly, when the samples were separated by gender, significant differences were observed between female MDD patients and normal females at the same site $(P<0.05$; Figure $2 C)$, while no significant differences were observed between the male groups (data not shown).

\section{A}

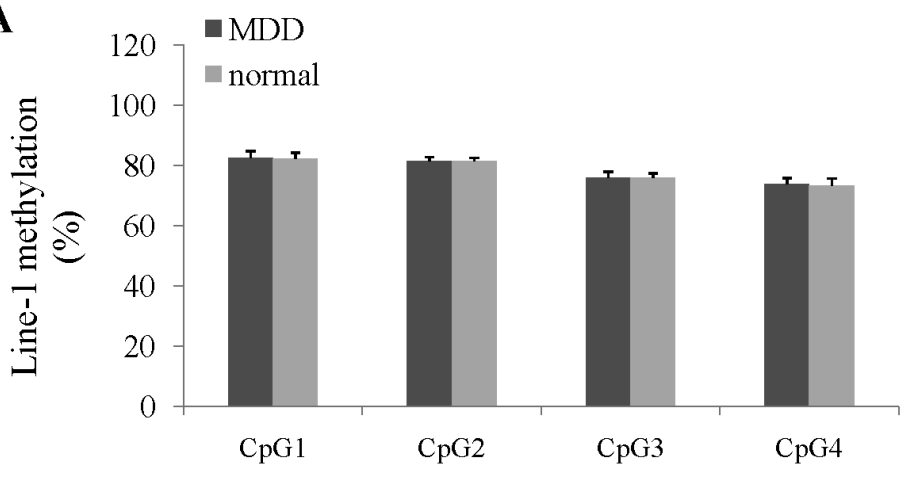

B

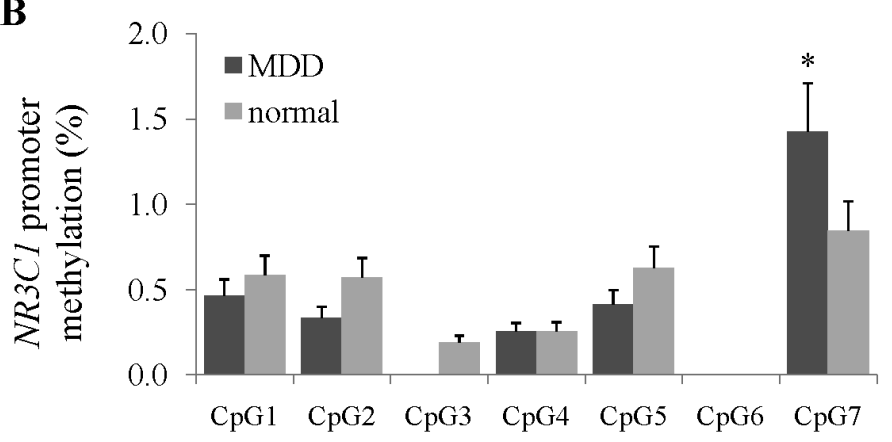

C

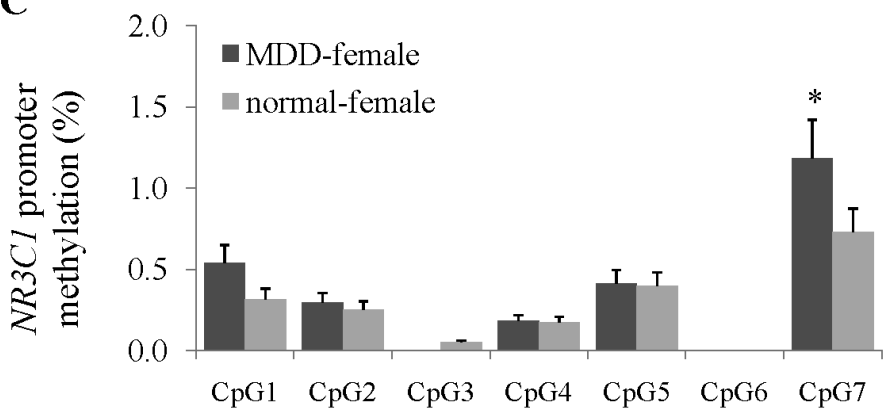

Figure 2. Percentages of methylation status (mean $\pm S E M)$ in Thai MDD patients $(N=29)$ and normal controls $(N=$ 33). A. LINE-1 methylation status at four CpG sites. B. Methylation status (mean \pm SEM) of seven $\mathrm{CpG}$ sites within a predicted NGFI-A binding site of the exon $1 \mathrm{~F} \mathrm{NR3C1} \mathrm{promoter} \mathrm{region.} \mathrm{C.} \mathrm{Methylation} \mathrm{status} \mathrm{of} \mathrm{the} \mathrm{similar} \mathrm{site} \mathrm{as} \mathrm{in} \mathrm{(B)}$ in female MDD patients $(\mathrm{N}=20)$ and normal Thai females $(\mathrm{N}=26) .{ }^{*} \mathrm{P}<0.05$. 


\section{NR3C1 expression in Thai MDD patients}

The expression levels ofNR3C1 were analyzed by real-time PCR on blood-derived cDNA. No significant difference were observed in the expression levels between samples from MDD patients and normal subjects $(P>0.05$; Figure 3$)$ and no significant differences were observed when the samples were separated by gender $(P>0.05$; Figure 3$)$.

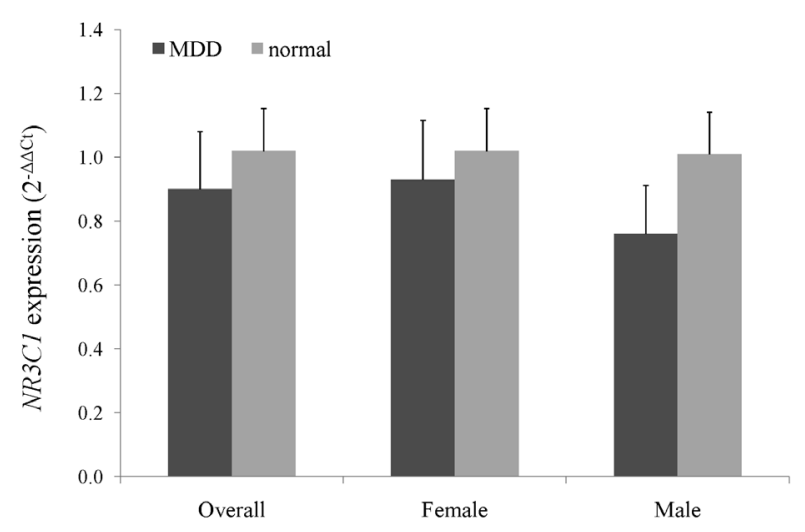

Figure 3. NR3C1 mRNA expression ratios between MDD patients and normal controls. The full sample set was derived from 33 MDD patients and 12 control subjects; female samples were derived from 23 MDD patients and 10 controls; male samples were derived from $10 \mathrm{MDD}$ patients and two controls.

\section{Cortisol levels in Thai MDD patients}

The cortisol levels in the blood plasma of MDD patients and control subjects were analyzed to determine the functional implications of $N R 3 C 1$ hypermethylation. The highest $(14.38 \mu \mathrm{g} / \mathrm{dL})$ and lowest $(4.42 \mu \mathrm{g} / \mathrm{dL})$ cortisol levels were observed in a depressed female and a normal female, respectively, where normal reference levels of cortisol are 8.7-22.4 $\mu \mathrm{g} / \mathrm{dL}$ (Peeters et al., 2003). No significant differences were observed in the cortisol levels of MDD patients compared with normal subjects ( $P$ > 0.05; Figure 4).

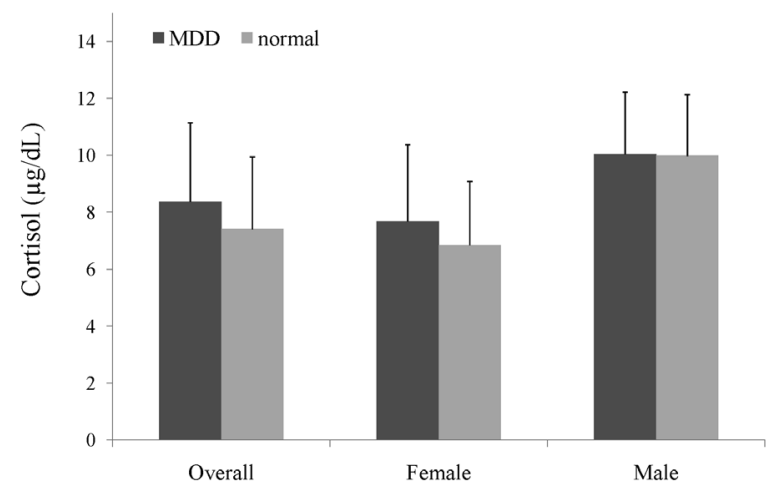

Figure 4. Cortisol levels in blood plasma samples from Thai MDD patients $(N=18)$ and normal controls $(N=12)(P>$ 0.05). The full sample set was derived from $18 \mathrm{MDD}$ patients and 2 control subjects; female samples were derived from 12 MDD patients and 10 controls; male samples were derived from six MDD patients and two controls. 


\section{DISCUSSION}

Methylation at a potential NGFI-A transcription factor binding site of the NR3C1 gene has been implicated in the regulation of HPA function in response to early environmental factors. According to the results of bisulfite-pyrosequencing of blood-derived samples at seven CpG sites in the NR3C1 promoter region in this study, significant differences $(P<0.05)$ in the mean methylation statuses between MDD patients and normal controls were only observed at CpG7 (Figure 2B). These findings are in agreement with a previously reported correlation between DNA methylation and stress disorders described by Oberlander et al. (2008) and McGowan et al. (2009). Interestingly, the methylation findings reported here also reveal significantly higher methylation levels at CpG7 in female MDD patients compared with normal females $(P<0.05$; Figure $2 C$ ). The NR3C1 hypermethylation results therefore suggest that the hypermethylation status at this specific site may positively correlate with depression disorder in Thai MDD patients, especially in females. A recent study similarly showed that an epigenetic risk factor of the ADCYAP1R1 gene, which was associated with an estrogen stress response element, has a higher impact on the later development of posttraumatic stress disorder (PTSD) in women than in men (Ressler et al., 2011). These findings may explain the higher rate of stress-related mood disorders in women compared with men. It has furthermore been suggested that clinical investigations of PTSD and depression should focus on sex differences in the epigenetic regulation of HPA-axis activity in response to stress and adverse events (Raabe and Spengler, 2013) and the findings of a recent study suggest that altered DNA methylation status may be used as a biomarker of depression (Song et al., 2014).

Based on the methylation analysis results in this study, we hypothesized that DNA methylation in the exon $1 \mathrm{~F}$ region of the $N R 3 C 1$ gene promoter may decrease $N R 3 C 1 \mathrm{mRNA}$ expression, thereby influencing the negative feedback mechanism in the HPA axis (de Kloet et al., 2005; McGowan et al., 2009); however, no significant differences in NR3C1 mRNA expression levels were observed between the MDD patients and control subjects. Although there was a trend of lower NR3C1 expression in MDD patients compared with controls, these apparent differences were not significant, possibly due to the low levels of NR3C1 expression in the blood (Turner et al., 2006) and that such small changes in gene expression cannot be detected by this method. Another explanation is that some CpGs reflect the gene expression level better than others in the promoter region of the same gene (Everhard et al., 2009).

In this study, the cortisol levels in blood plasma of all participants were investigated as they have been associated with some stress-related disorders and abnormalities in the HPA stress response activity (Young et al., 2001; Pariante et al., 2004; Oberlander et al., 2008). Young et al. (2001) demonstrated that $80 \%$ of examined MDD patients had lower serum cortisol levels than the standard level. On the other hand, no obvious difference in serum cortisol levels was observed between depressed male and healthy male subjects (Posener et al., 2004). Our findings reported here showed no significant differences in the cortisol levels in MDD patients compared with normal subjects, either in the whole sample set or separated by gender. Cortisol levels in all MDD samples were rather low, which is consistent with the low levels of serum cortisol reported for depression patients when blood samples were collected in the morning. The cortisol levels were observed to be higher in the afternoon (Burke et al., 2005).

In this study, the relationships between NR3C1 promoter methylation, NR3C1 gene expression, and plasma cortisol levels in MDD were assessed with aim of gaining insight into generelated causes of MDD. Hypermethylation was identified in the 7 th $\mathrm{CpG}$ of exon $1 \mathrm{~F}$ in the promoter 
region of the NR3C1 gene within a NGFI-A binding site in Thai MDD patients. No differences in NR3C1 mRNA expression and plasma cortisol levels were observed between the Thai MDD patients and the normal Thai control subjects. The findings reported here provide insight into the etiology and pathophysiology of MDD and others stress-related disorders.

\section{ACKNOWLEDGMENTS}

Research supported by grants from the Human Resource Development in Science Project (Science Achievement Scholarship of Thailand; SAST), the Center for Medical Genetics Research (CMGR), the Thailand Center of Excellence for Life Sciences (TCELS), and the 90th Anniversary of Chulalongkorn University Fund (Ratchadaphiseksomphot Endowment Fund).

\section{REFERENCES}

Bet PM, Penninx BW, Bochdanovits Z, Uitterlinden AG, et al. (2009). Glucocorticoid receptor gene polymorphisms and childhood adversity are associated with depression: New evidence for a gene-environment interaction. Am. J. Med. Genet. B Neuropsychiatr. Genet. 150B: 660-669.

Burke HM, Davis MC, Otte C and Mohr DC (2005). Depression and cortisol responses to psychological stress: a meta-analysis. Psychoneuroendocrinology 30: 846-856.

de Kloet ER, Joels M and Holsboer F (2005). Stress and the brain: from adaptation to disease. Nat. Rev. Neurosci. 6: $463-475$.

Everhard S, Tost J, El Abdalaoui H, Criniere E, et al. (2009). Identification of regions correlating MGMT promoter methylation and gene expression in glioblastomas. Neuro-Oncol. 11: 348-356.

Francke $U$ and Foellmer BE (1989). The glucocorticoid receptor gene is in 5q31-q32 [corrected]. Genomics 4: 610-612.

Hollenberg SM, Weinberger C, Ong ES, Cerelli G, et al. (1985). Primary structure and expression of a functional human glucocorticoid receptor cDNA. Nature 318: 635-641.

Howell MP and Muglia LJ (2006). Effects of genetically altered brain glucocorticoid receptor action on behavior and adrenal axis regulation in mice. Front. Neuroendocrinol. 27: 275-284.

Jacobson L and Sapolsky R (1991). The role of the hippocampus in feedback regulation of the hypothalamic-pituitaryadrenocortical axis. Endocr. Rev. 12: 118-134.

McGowan PO, Sasaki A, D'Alessio AC, Dymov S, et al. (2009). Epigenetic regulation of the glucocorticoid receptor in human brain associates with childhood abuse. Nat. Neurosci. 12: 342-348.

Meaney MJ (2001). Maternal care, gene expression, and the transmission of individual differences in stress reactivity across generations. Annu. Rev. Neurosci. 24: 1161-1192.

Moser D, Molitor A, Kumsta R, Tatschner T, et al. (2007). The glucocorticoid receptor gene exon 1-F promoter is not methylated at the NGF1-A binding site in the human hippocampus. World J. Biol. Psychiatr. 8: 262-268.

Oberlander TF, Weinberg J, Papsdorf M, Grunau R, et al. (2008). Prenatal exposure to maternal depression, neonatal methylation of human glucocorticoid receptor gene (NR3C1) and infant cortisol stress responses. Epigenetics 3: 97-106.

Pariante CM, Thomas SA, Lovestone S, Makoff A, et al. (2004). Do antidepressants regulate how cortisol affects the brain? Psychoneuroendocrinology 29: 423-447.

Peeters F, Nicholson NA and Berkhof $\mathrm{J}$ (2003). Cortisol responses to daily events in major depressive disorder. Psychosom. Med. 65: 836-841.

Perroud N, Paoloni-Giacobino A, Prada P, Olie E, et al. (2011). Increased methylation of glucocorticoid receptor gene (NR3C1) in adults with a history of childhood maltreatment: a link with the severity and type of trauma. Transl. Psychiatr. 1: e59.

Pfaffl MW (2001). A new mathematical model for relative quantification in real-time RT-PCR. Nucl. Acids Res. 29 : e45.

Posener JA, DeBattista C, Veldhuis JD, Province MA, et al. (2004). Process irregularity of cortisol and adrenocorticotropin secretion in men with major depressive disorder. Psychoneuroendocrinology 29: 1129-1137.

Raabe FJ and Spengler D (2013). Epigenetic risk factors in PTSD and depression. Front. Psychiatr. 4: 80.

Ressler KJ, Mercer KB, Bradley B, Jovanovic T, et al. (2011). Post-traumatic stress disorder is associated with PACAP and the PAC1 receptor. Nature 470: 492-497.

Song Y, Miyaki K, Suzuki T, Sasaki Y, et al. (2014). Altered DNA methylation status of human brain derived neurotrophis factor gene could be useful as biomarker of depression. Am. J. Med. Genet. B Neuropsychiatr. Genet. 165B: $357-364$.

Turner JD, Schote AB, Macedo JA, Pelascini LP, et al. (2006). Tissue specific glucocorticoid receptor expression, a role for 
alternative first exon usage? Biochem. Pharmacol. 72: 1529-1537.

Weaver ICG, Cervoni N, Champagne FA, D'Alessio AC, et al. (2004). Epigenetic programming by maternal behavior. Nat. Neurosci. 7: 847-854.

World Health Organization (2012). Depression: AGlobal Crisis. World Mental Health Day, October 10. Geneva. Available at [http://www.unis.unvienna.org/unis/pressrels/2012/unissgsm378.html]. Accessed August 21, 2014.

Yang AS, Estecio MRH, Doshi K, Kondo Y, et al. (2004). A simple method for estimating global DNA methylation using bisulfite PCR of repetitive DNA elements. Nucl. Acids Res. 32: e38.

Young EA, Carlson NE and Brown MB (2001). Twenty-four-hour ACTH and cortisol pulsatility in depressed women. Neuropsychopharmacology 25: 267-276. 